F. ROUZAUD, J. MARTIN, F. GALLET,

D. DELOURME, J.-M. PETIT, H. LEVÉZIEL, R. JULIEN, A. OULMOUDEN

INRA / Université de Limoges, UMR de Génétique moléculaire animale, Faculté des Sciences, 123 avenue Albert Thomas, 87060 Limoges cedex
7 - Utilisation des marqueurs génétiques

\section{Utilisation de marqueurs génétiques pour la traça- bilité. Intérêt des gènes de la coloration de la robe chez le bovin}

Résumé. Les marqueurs génétiques, qu'ils soient anonymes (microsatellites) ou responsables de phénotypes (gènes de la coloration, par exemple), constituent des outils efficaces pour identifier les individus ou les populations (races) et pour assurer leur traçabilité. On montre ici l'intérêt du gène extension pour le génotypage des bovins.

La traçabilité des produits d'origine animale est une question de grande actualité depuis que des agents de nouvelles maladies (ESB), des substances prohibées (hormones) ou des contaminants chimiques (dioxine) ont été découverts dans les viandes notamment. Afin d'assurer sa sécurité alimentaire, le consommateur souhaite connaître l'origine (la race, le lieu et les conditions d'élevage, etc) et les transformations apportées aux produits animaux destinés à la boucherie.

A cet effet, l'utilisation de marqueurs génétiques peut fournir des méthodes efficaces pour identifier les individus ou les populations animales et mieux assurer leur traçabilité.

\section{1 / Identification des individus}

Lidentification des individus repose sur l'utilisation de marqueurs ADN microsatellites constitués de la répétition, variable selon les individus, de deux ou quelques nucléotides en un lieu particulier (locus) du génome. Pour une espèce donnée, plusieurs milliers de tels loci sont aléatoirement distribués dans le génome. En choisissant judicieusement les microsatellites c'est-à-dire en combinant leur nombre et le polymorphisme de leurs tailles, des profils d'identification propres à chaque individu d'une espèce pourront être obtenus. De telles méthodes sont développées en France par LABOGENA (Jouy-en-Josas) dans une optique de traçabilité individuelle des animaux et produits dérivés (viandes notamment).

\section{2 / Identification des populations}

En principe, les microsatellites pourraient être également utilisés pour identifier des groupes d'individus correspondant à des « races » ou des populations animales sélectionnées et plusieurs équipes du Département INRA de Génétique animale s'y emploient également. Dans la pratique, la difficulté réside dans la recherche des bons loci discriminateurs de " races ». Les microsatellites ne sont pas des gènes codants (ce sont des séquences dites anonymes) et ne peuvent pas être attribuables à un caractère défini (un phénotype). A l'inverse, on peut s'intéresser à des gènes responsables d'un phénotype racial et, à la condition qu'ils soient suffisamment polymorphes, la combinatoire de leurs allèles respectifs est susceptible de fournir une grille d'identification des populations.

\section{1 / Les gènes de la coloration}

C'est le cas des gènes de la coloration de la robe chez les bovins. On considère aujourd'hui que les processus cellulaires et biochimiques responsables de la pigmentation sont sous la dépendance d'une centaine de gènes (Urabe et al 1993) parmi lesquels une dizaine auraient une importance majeure (tableau 1). Les produits de certains gènes contrôlent des fonctions aux niveaux tissulaire ou cellulaire : c'est le cas, par exemple, des gènes codant pour les récepteurs mélanoblastiques $c-k i t, E T_{A}-R, E T_{B}-R$, et leurs ligands. Ils interviennent dans la survie/prolifération des cellules précurseurs des mélanocytes 
et leur migration à partir de la crête neurale vers les couches de l'épiderme. D'autres gènes contrôlent la mélanogenèse proprement dite (synthèse intramélanocytaire des différents types de pigments : eumélanines noires et phaeomélanines rouges ) : c'est le cas des gènes codant pour la tyrosinase et les protéines associées TRP1 et TRP2. D'autres encore contrôlent le routage intracellulaire des mélanosomes (organites sièges de la mélanogenèse) depuis la région centrale de la cellule vers les extrémités dendritiques. C'est le cas de la myosine Va, véritable moteur moléculaire, produit du gène dilute, qui assure le mouvement des mélanosomes le long des fibres protéiques du cytosquelette.

Tableau 1. Gènes majeurs impliqués dans la coloration de la robe chez les bovins.

\begin{tabular}{|c|c|c|c|}
\hline Gène & $\begin{array}{l}\text { Nom du locus } \\
\text { correspondant } \\
\text { (chez la souris) }\end{array}$ & Fonction de la protéine & Phénotype \\
\hline c-kit & dominant spotting & récepteur du ménaloblaste & pie \\
\hline steel & steel & ligand du récepteur c-kit & pie \\
\hline$E T_{A}-R$ & - & récepteur du ménaloblaste & pie \\
\hline$E T_{B^{-}} R$ & - & récepteur du ménaloblaste & pie \\
\hline ET1 & endothéline & ligand de $E T_{A}-R$ et de $E T_{B}-R$ & pie \\
\hline$M C 1-R$ & extension & récepteur mélanocytaire de $\alpha$-MSH & coloration rouge/noire \\
\hline POMC & $P O M C$ & $\alpha-\mathrm{MSH}$, ligand agoniste de MC1-R & noir \\
\hline agouti & agouti & ligand antagoniste de MC1-R & rouge \\
\hline dilute & dilute & Myosine Va, transport des mélanosomes & intensité de la coloration \\
\hline $\operatorname{tyr}$ & tyr & enzyme majeure de la mélanogénèse & présence d'une coloration \\
\hline$T R P 1$ & brown & enzyme associée à la tyrosinase & modulation de la coloration \\
\hline$T R P 2$ & slaty & DOPAchrome tautomérase & modulation de la coloration \\
\hline
\end{tabular}

\section{2 / Le gène extension}

Le gène extension code pour le récepteur mélanocytaire (MC1-R) de l'hormone $\alpha$-MSH (« melanocyte-stimulating hormone »). Ce récepteur appartient à la grande famille des récepteurs couplés aux protéines G. Par son interaction, l'hormone stimule une voie de transduction du signal favorable à l'expression des gènes de la mélanogenèse et à la synthèse des eumélanines noires. Un deuxième ligand du récepteur, le produit du gène agouti, « antagonise » l'action de l'hormone et favorise par un mécanisme encore mal compris la synthèse des phaeomélanines. Chez les mammifères, plusieurs allèles du gène extension ont été récemment identifiés (Klungland et al, 1995) et, selon la structure du récepteur, associés à des phénotypes de coloration variable dans les gammes du noir et du rouge.

\section{3 / Un test de génotypage}

La caractérisation dans les races bovines de quatre allèles du gène extension $\left(E, e, E_{1}, E^{D}\right)$ a débouché récemment sur la mise au point d'un test de génotypage (Oulmouden et al 1998). Il est basé sur la découverte d'un allèle du gène extension associé à des phénotypes de coloration rouge ou noire fortement atténuée (races Aubrac et Gasconne). Nommé pour l'instant $E_{1}$, cet allèle se caractérise, comparativement à l'allèle de référence $E$, par une duplication de 12 nucléotides, soit 4 acides aminés surnuméraires dans la troisième boucle intra-cytoplasmique du récepteur (boucle associée à la protéine $\mathrm{G}$ ). On suppose que cette modification structurale altère le contact avec la protéine G et atténue le signal engendré par ce type de récepteur. Les deux autres allèles mutants sont $e$ (où la délétion d'une guanine change le cadre de lecture et conduit à un récepteur tronqué ayant perdu le site de liaison de l'hormone $\alpha$-MSH) et $E^{D}$ (où la substitution d'une thymine par une cytosine aboutit au remplacement d'une leucine par une proline, ce qui rend le récepteur constitutionnellement actif et engendre le phénotype noir dominant). Ces divers polymorphismes moléculaires conduisent à l'apparition-disparition de sites de clivage par les enzymes Aci I et Bsrf I de sorte qu'avec un choix approprié d'amorces PCR, on obtient des amplifiats que l'on peut ou non cliver par les endonucléases citées. En combinant les profils électrophorétiques obtenus (figure 1), on détermine directement pour chaque individu examiné la formule allélique du gène extension. On trouvera dans le tableau 2 des exemples de génotypes rencontrés chez quelques centaines d'individus appartenant à diverses races bovines françaises.

Une assez bonne discrimination entre les races laitières (allèles $E$ et $E^{D}$ ) et les races allaitantes (allèles $e$ et $E_{1}$ ) peut être constatée. La découverte de nouveaux allèles du gène extension ou, mieux encore, l'exploitation du polymorphisme d'autres gènes de la coloration comme dilute par exemple, devrait favoriser la construction d'un arbre de décision conduisant à une identification fine des diverses populations. On peut envisager que ce type de protocole s'applique à d'autres espèces de rente. En effet, chez les vertébrés, le déterminisme génétique de la coloration de la peau et des phanères semble être sous la dépendance d'un ensemble de gènes homologues. 
Tableau 2. Génotype pour les gènes extension dans quelques races bovines françaises.

\begin{tabular}{|l|c|c|c|c|c|c|}
\hline Race & \multicolumn{5}{|c|}{ Génotype } \\
& e/e & e/E1 & E1/E1 & E/E1 & E/E & E $^{\mathrm{D}} / \mathrm{E}^{\mathrm{D}}$ \\
\hline Aubrac & 1 & 2 & 42 & 17 & 6 & \\
Gasconne & & 23 & 23 & 18 & & \\
Salers & 56 & & & & & \\
Blonde & 27 & & & & & \\
Limousine & 42 & & & & & \\
Charolaise & 59 & & & & 75 & \\
Normande & & & & & 75 & 87 \\
Prim'Holstein & & & & & & 87 \\
\hline
\end{tabular}

\section{Références}

Kungland H., Vage D.I., Gomez-Raya L., Adalsteinsson S., Lien S., 1995. The role of melanocyte-stimulating hormone (MSH) receptor in bovine coat color determination. Mammalian Genome, 6, 636-639.

Oulmouden A. et al, 1998. Procédé d'identification de l'origine génétique de populations bovines françaises. Demande de brevet français $\mathrm{n}^{\circ} 9805809$ (7 mai 1998), INRA/Université de Limoges.

Urabe K., Aroca P., Hearing V.J., 1993. From gene to protein : determination of melanin synthesis. Pigment Cell Research, 6, 186-192.K

Figure 1. Principe du test de génotypage des races bovines françaises, utilisant le polymorphisme du gène extension (présence ou non de sites de clivage pour Aci I et/ou Bsrf I; présence ou non d'une duplication de 12 pb (allèle E1).
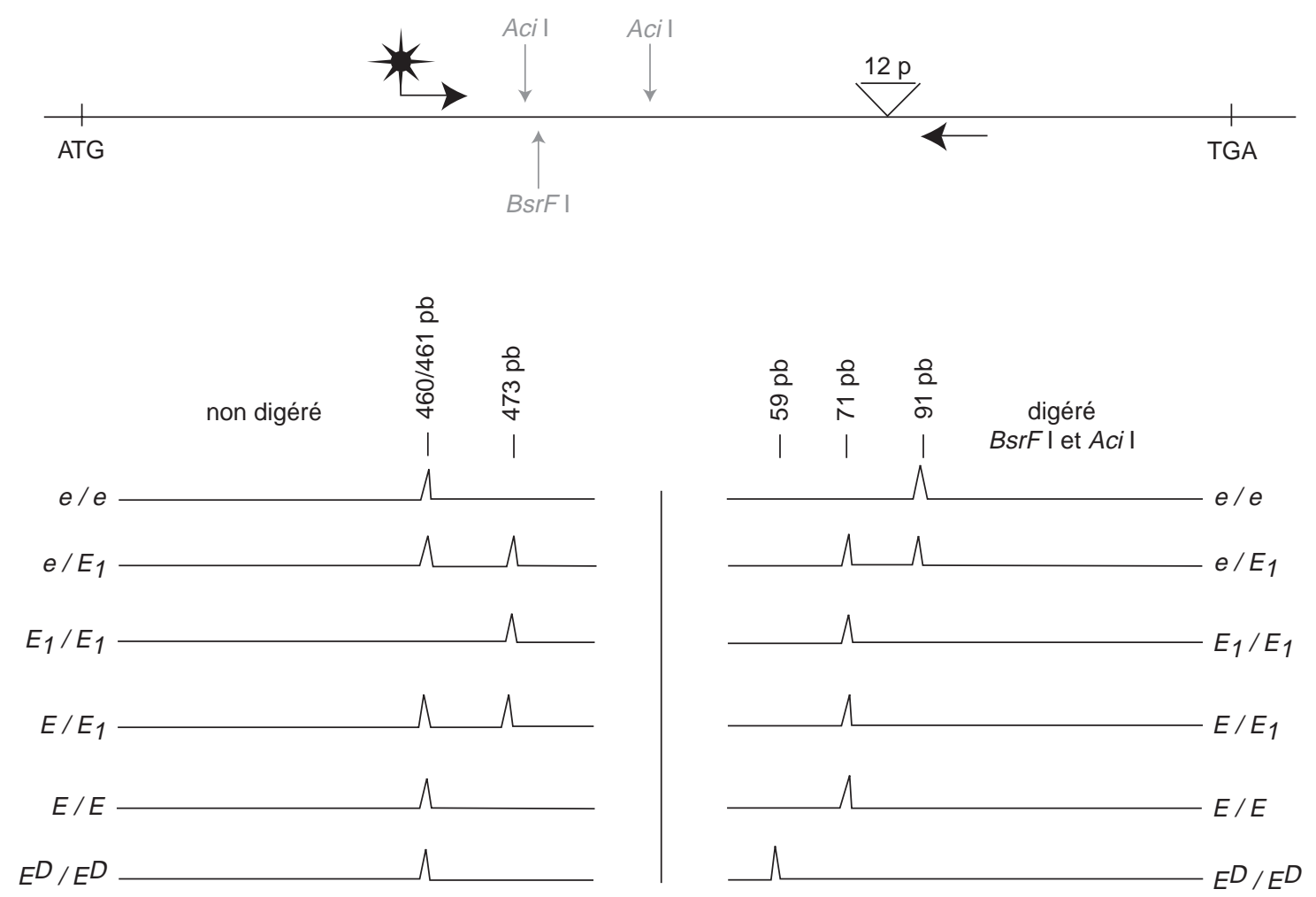